\title{
Preface: Selected Papers from the Workshop Bioinformatics and Artificial Intelligence Joined with the International Joint Conference on Artificial Intelligence
}

\author{
ABDOULAYE BANIRÉ DIALLO ${ }^{1}$ ENGELBERT MEPHU NGUIFO, ${ }^{2}$ and MOHAMMED J. ZAKI ${ }^{3}$
}

T HIS VOLUME CONTAINS a selected subset of the articles presented at the two first editions of the workshop Bioinformatics and Artificial Intelligence (BAI) joined with the International Joint Conference on Artificial Intelligence. The format of the workshops was one-day format with one keynote speaker, two invited speakers, and seven oral presentations. The goal of BAI is to bring together active scholars and practitioners at the frontiers of artificial intelligence (AI) and bioinformatics. AI holds a tremendous repertoire of algorithms and methods that constitute the core of different topics of bioinformatics and computational biology research. BAI goals are twofold: (1) How can AI techniques contribute to bioinformatics research? and (2) How can bioinformatics research raise new fundamental questions in AI? Contributions clearly point out answers to one of these goals focusing on AI techniques as well as focusing on biological problems.

The first edition was held in Buenos Aires, Argentina on July 27th, 2015. The second edition was held in New York, United States, on July 11th, 2016. The two workshops were chaired by Abdoulaye Baniré Diallo, Engelbert Mephu Nguifo, and Mohammed J. Zaki. During the two editions, the articles submitted to the workshops were carefully peer reviewed by at least three members of the program committee and 14 articles with the highest scores were selected (7 articles each edition). Accepted articles were then invited for submission to a special issue of the Journal of Computational Biology. The submissions to the special issue went through an additional round of reviewing before publication. We would like to thank all the PC members and the reviewers for their reviews, as well as all the authors for their contributions, Angel Murakami, for the smooth cooperation regarding various organization details, and Sorin Istrail and the editorial staff at Journal of Computational Biology for numerous discussions, helpful tips, and technical support.

\section{Address correspondence to: Abdoulaye Baniré Diallo Department of Computer Science Université du Québec à Montréal PO BOX 8888 Downtown station Montreal, Quebec, H3C $3 P 8$ \\ Canada \\ E-mail: diallo.abdoulaye@uqam.ca}

\footnotetext{
${ }^{1}$ Department of Computer Science, Université du Québec à Montréal, Montreal, Canada.

${ }^{2}$ Université Clermont Auvergne, Clermont-Ferrand, Laboratoire d'Information de Modélisation, et Optimisation des Systèmes (LIMOS), Aubière cedex, France.

${ }^{3}$ Department of Computer Science, Rensselaer Polytechnic Institute, Troy, New York.
} 